\title{
Association of tumor size and extrathyroidal extension with local relapse in differentiated thyroid cancer in the mexican population
}

\author{
Javier López-Gómez ${ }^{1 *}$, Martín Granados-Garciaª , E. Alejandra Palafox-Parrilla², José Granel/ and \\ Raimundo Gutiérrez-Fonseca ${ }^{3}$
}

${ }^{1}$ Head \& Neck Department, Instituto Nacional de Cancerología, Mexico City, México; ${ }^{2}$ Epidemiology Department, National Coordination of Cancer Prevention and Control Programs, Instituto Nacional de Cancerología, Mexico City, Mexico; ${ }^{3}$ Otorhinolaryngology Department, Hospital Universitario Rey Juan Carlos, Madrid, Spain

\begin{abstract}
Introduction: Thyroid cancer is the second neoplasm with higher incidence in Mexican women. Its low mortality indicates that it could be treated with hemithyroidectomy without sacrificing cure, our objective was to find a tumor size associated with a risk of local relapse to recommend this procedure. Material and methods: We studied a retrospective cohort of 278 cases, and selected 91 patients for a matched pair analysis. There were 14 cases treated with hemithyroidectomy alone and they were compared with 77 controls treated with total thyroidectomy and previously paired for the main prognostic factors. The relationship between tumor size and extrathyroid extension was studied. Also the tumor size and the extension of the surgery with relapse. Results: Tumor size greater than $2 \mathrm{~cm}$ was associated with a RR 5.5 of presenting extrathyroidal macroscópica extension, $19 \%$ of patients had second malignancies. Conclusions: There is a relationship between extrathyroidal extension and tumor size. Patients with tumor size less than $2 \mathrm{~cm}$ and treated with hemithyroidectomy alone were not distinct in relapse rate when compared with those treated with total thyroidectomy. Therefore, hemithyroidectomy can be appropriate for selected patients with tumors smaller than $2 \mathrm{~cm}$.
\end{abstract}

Key words: Lobectomy. Extrathyroidal extension. Differentiated thyroid cancer. Local recurrence. Tumor size.

\section{Introduction}

There is controversy regarding the best surgical management of differentiated thyroid carcinoma. Some support systematic total thyroidectomy, since it would facilitate iodine ablation and relapse or persistence detection; in addition, morbidity is minimal in centers with experience; however, others favor lobectomy in order to reduce total thyroidectomy-associated morbidity such as permanent hypoparathyroidism in selected cases, as well as to decrease thyroid hormones' administration in a large percentage of patients, although a higher risk of relapse in the remaining thyroid lobe (14\%) has been documented due to papillary carcinoma frequent multi-centrality, which reaches up to $40 \%$. However, with an appropriate patient selection, the relapse rate has been documented to be as low as 1 to $4 \%$, with complementary thyroidectomy rates being lower than $10 \%$, without survival being affected ${ }^{1-6,12}$.

The tumor size total thyroidectomy is recommended with has been controversial, with a range of 1 to $4 \mathrm{~cm}$. However, in the main treatment guidelines, total thyroidectomy is recommended in patients with tumors larger than $4 \mathrm{~cm}$, with macroscopic extrathyroidal extension
Correspondence:

*Javier López-Gómez

E-mail: dr.javierlopgom@gmail.com

DOI: 10.24875/j.gamo.M19000193
Date of reception: 07-08-2019

Date of acceptance: 27-09-2019
Available online: 11-11-2019 Gac Mex Oncol. 2020;19(1):5-12 www.gamo-smeo.com 1665-9201/@ 2019 Sociedad Mexicana de Oncología. Published by Permanyer. This is an open access article under the terms of the CC BY-NC-ND license (http://creativecommons.org/licenses/by-nc-nd/4.0/). 
or in case of evidence of regional or distant disease ${ }^{8}$, although a $1-\mathrm{cm}$ size had been previously proposed for resection. This reflects how poorly has this issue been studied. We believe that increasing tumor size is associated with a higher risk of macroscopic extrathyroidal extension. This is important, since it determines a risk of local relapse that jeopardizes patient survival. On the other hand, this is different from contralateral lobe local relapse when lobectomy is performed, which is regularly rescued with a new surgery without an impact on long-term survival. We believe that tumor size has not been sufficiently analyzed as an indicator of surgical appropriate extent, and this is why we undertook this study.

\section{Objective}

To determine which tumor size is associated with a low risk of macroscopic extrathyroidal extension in order to recommend thyroid lobectomy.

\section{Methodology}

Approval by the Ethics and Research Committee of the National Institute of Cancer (INCan - Instituto Nacional de Cancerología) with registry number 0060 was obtained, and the records of patients newly diagnosed with differentiated thyroid cancer who were admitted to the INCan from September 2006 to September 2016 were reviewed. Seven-hundred and forty-nine cases were identified, but only those patients fully treated at INCan, with corroborated diagnosis of papillary carcinoma or follicular carcinoma and complete records were included in the retrospective cohort. Patients with different diagnoses, or who were treated outside the INCan, or who had incomplete records were excluded. The final sample to be analyzed comprised 278 patients.

The following independent variables were analyzed: age, gender, tumor size $(\mathrm{T})$, lymph node status $(\mathrm{N})$, presence of metastasis $(\mathrm{M})$, histological variants, multi-centrality, perineural dissemination, lymphovascular permeation, follow-up, surgery extension, clinical stage according to the American Joint Committee on Cancer (AJCC) 2018 criteria and risk group according to the American Thyroid Association (ATA) 2015 criteria, prior radiotherapy to the neck, family history of thyroid cancer and presence of other primary tumors. And as dependent variables, extrathyroidal extension and relapse were analyzed.

Extrathyroidal extension was classified as: a) absent, when the tumor was limited to the capsule, without extension; b) microscopic, when the tumor compromised the capsule and invaded perithyroidal fat, but without compromising adjacent structures, and c) gross or macroscopic, when the tumor compromised the capsule and extended to adjacent structures (muscle, recurrent laryngeal nerve, trachea, larynx, esophagus, pharynx, blood vessels or soft tissue).

Relapse was classified according to ATA criteria (2015) as: a) biochemical relapse, if thyroglobulin (Tg) levels were higher than $0.2 \mathrm{ng} / \mathrm{mL}$ with suppressed thyroid-stimulating hormone (TSH), or higher than $1 \mathrm{ng} / \mathrm{mL}$ with stimulated TSH and in the absence of anti-Tg antibodies; b) local relapse, in the presence of macroscopic tumor in the tumor bed on clinical examination or by imaging, corroborated by fine-needle aspiration biopsy (FNAB), or documented in the pathology final report; c) regional relapse, in the presence of macroscopic tumor in lymph chains on clinical examination or by imaging and corroborated by FNAB or in the pathology final report, and d) distant relapse, with evidence of metastatic activity by computed axial tomography, positron-emission tomography (PET/CT) or iodine uptake test 4 months after treatment. Different relapse combinations were analyzed.

With the 278 patients who initially met the inclusion criteria, a nested case-control study was conducted. Sample size calculation was by convenience, and all 14 cases undergoing lobectomy and 77 controls treated with total thyroidectomy were included and were matched by the main prognostic factors according to AJCC 2018 and ATA 2015 criteria in order to control for confounding factors associated with relapse (i.e., age, absence of lymph node or distant metastases and absence of obvious macroscopic extrathyroidal extension within the preoperative or transoperative period).

Data were analyzed, with the association of tumor size and extrathyroidal extension with relative risk being measured, as well as that of tumor size and extent of surgery with the risk of relapse, by applying the chisquare test, with a 95\% confidence interval (Fig. 1). Statistical significance was assumed with a $p$-value lower than 0.05 .

\section{Results}

Two-hundred and seventy-eight patients were identified: 38 men (13.67\%) and 240 women (86.33\%), at a 6:1 ratio. Average follow-up was 60 months, with a range from 300 to 3,751 days. Sixty-six patients were older than 55 years $(23.74 \%)$ and 212 were younger than 55 years $(76.26 \%)$. After a mean follow-up of 


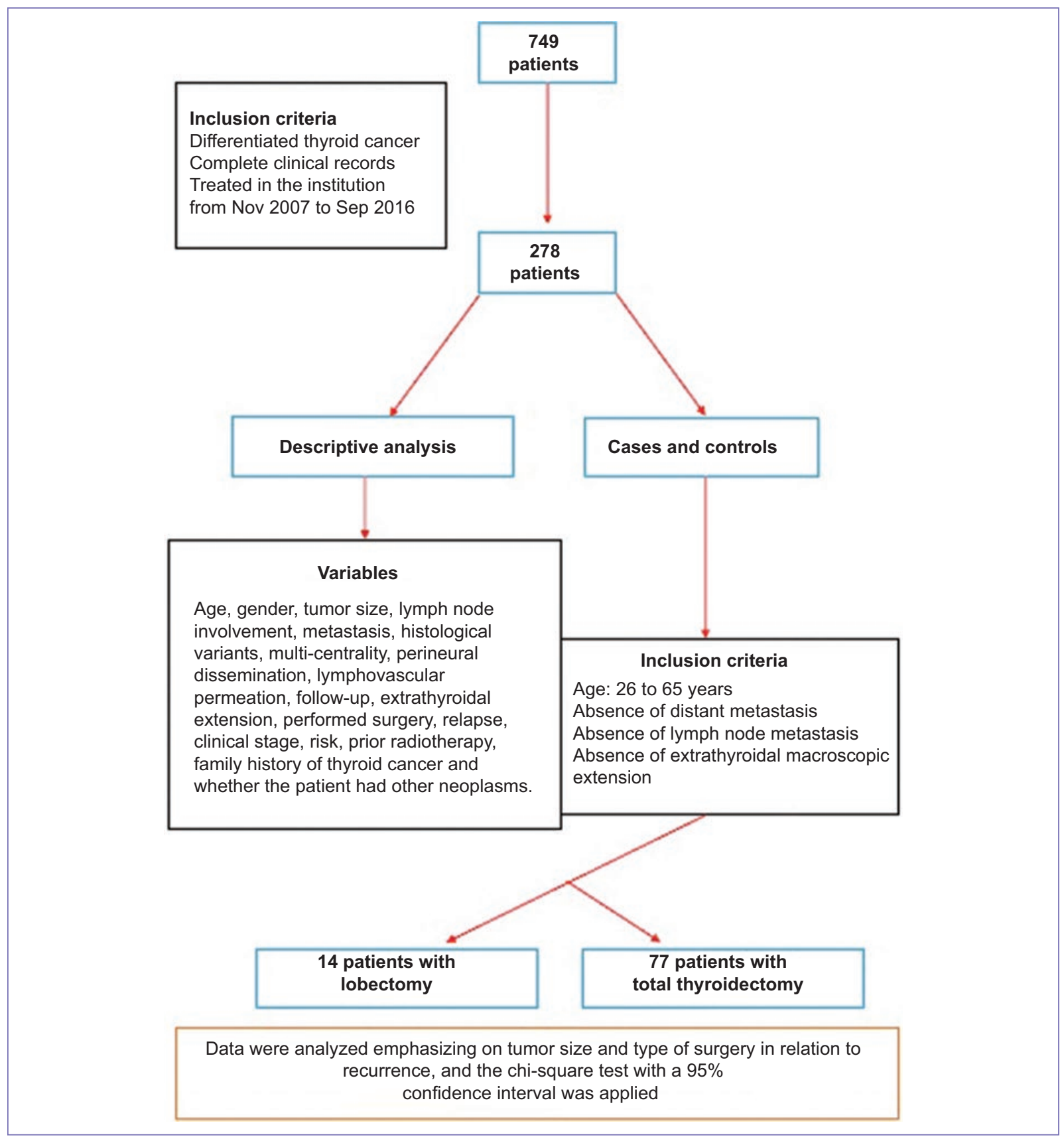

Figure 1. Methodology algorithm.

5 years, 199 patients $(71.58 \%)$ were disease-free and $79(28.42 \%)$ had persistence or relapse. Regarding relapses, $15.18 \%$ were biochemical, $12.65 \%$ local structural and $72.15 \%$ were regional structural or distant. Only two patients had died with tumor activity; given that this is a neoplasm with low mortality, no overall survival analysis was performed (Table 1).

According to the ATA 2015 criteria, 36 patients $(12.95 \%)$ were classified at low risk, $106(38.13 \%)$ at intermediate risk and 136 patients (48.92\%) were classified as being at high risk. Relapse in relation to risk groups showed a relapse rate of $36.76 \%$ among high-risk patients, out of which biochemical relapse accounted for $6.61 \%$, local relapse for $3.67 \%$ and distant or regional relapse for $26.47 \%$. In the intermediate risk group, the relapse rate was $24.52 \%$ : biochemical in $2.83 \%$, local in $3.77 \%$ and regional or distant in $17.92 \%$. In the low risk group, relapse was $8.33 \%$, with 
Table 1. Type of recurrence and grouped recurrence

\begin{tabular}{|c|c|c|c|}
\hline \multicolumn{2}{|c|}{ Type of recurrence } & \multicolumn{2}{|c|}{ Grouped recurrence } \\
\hline Type of recurrence & Patients & & \\
\hline Biochemical & $12(4.31 \%)$ & & \\
\hline Distant & $30(10.79 \%)$ & & \\
\hline Local & $4(1.43 \%)$ & Biochemical & $12(4.31 \%)$ \\
\hline Local and distant & $3(1.07 \%)$ & Local (all types) & $10(3.56 \%)$ \\
\hline Local and regional & $1(0.35 \%)$ & Others (regional and distant) & $57(20.50 \%)$ \\
\hline Local, distant and regional & $2(0.71 \%)$ & & \\
\hline Regional & $16(5.75 \%)$ & & \\
\hline Regional and distant & $11(3.95 \%)$ & & \\
\hline No recurrence & $199(71.58 \%)$ & & \\
\hline
\end{tabular}

no biochemical relapses, but local relapse was $2.77 \%$ and regional or distant relapse, $5.55 \%$. In other words, absence of disease (AD) in the low risk group was $91.66 \%$, in the intermediate risk group it was $75.47 \%$ and in the high risk group, $63.2 \%$. According to the AJCC 2018 criteria, 212 patients (76.26\%) were at stage I, 56 patients $(20.14 \%)$, at stage II, $4(1.44 \%)$, at stage III and $6(2.16 \%)$ were at metastatic stage.

At the discretion of the attending physician, 15 patients $(5.39 \%)$ were treated with lobectomy, 151 (54.31\%), with total thyroidectomy, 105 (37.76\%), with total thyroidectomy plus neck dissection and $7 \mathrm{pa}-$ tients $(2.51 \%)$ were treated with extended total thyroidectomy, with the latter group representing locally advanced cancer with invasion of adjacent structures.

The most common histology was conventional papillary cancer in 202 subjects (72.66\%), 49 (17.62\%) had papillary carcinoma with some poor-prognosis variant, 16 (5.75\%) had microcarcinoma and in 11 (3.95\%) follicular carcinoma was diagnosed.

Twelve patients (4.3\%) had a hereditary-family history of thyroid cancer, 15 (5.42\%) had previously received radiotherapy to the neck, and 53 patients (19.06\%) had second primary tumors, among which breast cancer accounted for $37.03 \%$, cervical cancer for $13.2 \%$, non-Hodgkin lymphoma for $7.54 \%$, non-melanoma skin cancer and endometrial cancer for $5.66 \%$ and follicular lymphoma and retroperitoneal sarcoma for $3.77 \%$ each. In addition, the following cancers occurred in $1.88 \%$ of patients: renal, parotid, lymphoma originating from mucosa-associated lymphoid tissue (MALT), oral cavity, prostate, ovarian, lung sarcoma, leukemia, malignant peripheral nerve sheath tumor, melanoma, esophageal and lymphoblastic lymphoma.

Fifty-two out of 278 patients showed no extrathyroidal extension (18.71\%), 155 (55.76\%) had microscopic extension and 71 (25.54\%) had macroscopic extension; 39 patients had tumors from 0 to $1 \mathrm{~cm}(14.02 \%), 84 \mathrm{had}$ tumors from 1 to $2 \mathrm{~cm}$ (30.21\%), 94 patients had tumors from 2 to $4 \mathrm{~cm}(33.81 \%)$ and 61 patients had tumors larger than $4 \mathrm{~cm}(21.94 \%)$.

When macroscopic extrathyroidal extension in relation to tumor size was analyzed, the risk of macroscopic extrathyroidal extension was found to be significantly increased from the 2 to $4-\mathrm{cm}$ group on, which included 33 patients $(35.5 \%)$, followed by $25(41 \%)$ in the group with tumors larger than $4 \mathrm{~cm}$, and by 11 patients (12.9\%) in the 1 to $2-\mathrm{cm}$ group and 1 patient (2.6\%) in the 0 to $1-\mathrm{cm}$ group.

Microscopic extrathyroidal extension was found in 59 patients $(38.06 \%)$ in the 1 to $2-\mathrm{cm}$ group, in $49(31.61 \%)$ in the 2 to $4-\mathrm{cm}$ group, in $30(19.35 \%)$ in the $>4-\mathrm{cm}$ group and in $17(10.96 \%)$ in the 0 to $1-\mathrm{cm}$ group. No macroscopic extrathyroidal extension was documented in the 0 to $1-\mathrm{cm}$ group, with 21 patients $(40.38 \%)$, as neither was in 13 subjects $(25 \%)$ of the 1 to $2-\mathrm{cm}$ group, $12(23.07 \%)$ in the 2 to $4-\mathrm{cm}$ group and in 6 patients $(11.53 \%)$ with tumors $>4 \mathrm{~cm}$.

The largest number of patients with extrathyroidal extension was observed in the 2 to $4-\mathrm{cm}$ group, with 93 patients (33.5\%); more in detail, macroscopic extrathyroidal extension occurred in $2.6 \%$ subjects in the 0 to $1-\mathrm{cm}$ tumors group, in $14.9 \%$ in the 1 to $2-\mathrm{cm}$ group, in $35.5 \%$ in the 2 to $4-\mathrm{cm}$ group and in $40.1 \%$ in the group of tumors larger than $4 \mathrm{~cm}$. A relative risk for 
Table 2. Tumor size and extrathyroidal extension

\begin{tabular}{|c|c|c|c|c|c|}
\hline Tumor size & 0 to $1 \mathrm{~cm}$ & 1 to $2 \mathrm{~cm}$ & 2 to $4 \mathrm{~cm}$ & $>4 \mathrm{~cm}$ & Total \\
\hline No extension/microscopic & $\begin{array}{c}38 \\
(97.4 \%)\end{array}$ & $\begin{array}{c}74 \\
(87.1 \%)\end{array}$ & $60(64.5 \%)$ & $\begin{array}{c}36 \\
(59 \%)\end{array}$ & $208(74.8 \%)$ \\
\hline Macroscopic extension & $\begin{array}{c}1 \\
(2.6 \%)\end{array}$ & $\begin{array}{c}11 \\
(12.9 \%)\end{array}$ & $33(35.5 \%)$ & $\begin{array}{c}25 \\
(41 \%)\end{array}$ & $70(25.2 \%)$ \\
\hline Total & $\begin{array}{c}39 \\
(14 \%)\end{array}$ & $\begin{array}{c}85 \\
(30.6 \%)\end{array}$ & $\begin{array}{c}93 \\
(33.5 \%)\end{array}$ & $\begin{array}{c}61 \\
(21.9 \%)\end{array}$ & 278 \\
\hline
\end{tabular}

Pearson's Chi-square test $=30.674$. $\mathrm{p}=0.000$.

macroscopic extrathyroidal extension of 5.5 was calculated in tumors larger than $2 \mathrm{~cm}$, which was statistically significant (95\% Cl: 2.8-10.9) (Tables 2 and 3).

Tables 4 to 6 and figures 2 and 3 show the analysis restricted to the 91 patients matched by main prognostic factors. In this cohort, there was no relationship between the extent of surgery and the type of relapse $(p=0.661$ ) (Table 4) (Fig. 2). Neither was an association found between the type of relapse and tumor size $(p=0.537)$ (Table 5) (Fig. 3), or between the extent of surgery and tumor size $(p=0.437)$ (Table 6$)$.

\section{Discussion}

Differentiated thyroid carcinoma is currently the second neoplasm with the highest incidence among Mexican women, after breast cancer $^{13}$, but despite its increasing incidence, mortality remains low and constant, which suggests that there is over-treatment of a relatively indolent neoplasm; therefore, we believe that selected cases could be less aggressively treated in order to avoid inappropriate morbidity, such as permanent hypoparathyroidism, as well as to decrease the need for taking thyroid hormones and recurrent laryngeal nerve lesions, without sacrificing the probability of long-term survival.

In our series, we found a higher proportion of patients younger than 55 years and females in comparison with data published by other authors (4:1), with this representing a group with better survival prognosis, where an effective treatment and lower associated morbidity is desirable.

As it is to be expected, we found that conventional papillary carcinoma was the most common histological type, followed by papillary carcinoma variants with poor prognosis, microcarcinomas and, less frequently, follicular carcinoma, which is consistent with published observations in this regard. We found an elevated rate of
Table 3. Risk estimate

\begin{tabular}{|l|c|c|c|}
\hline \multicolumn{3}{|c|}{ Risk estimate } \\
\hline \multicolumn{2}{|c|}{} & \multicolumn{2}{|c|}{$\begin{array}{c}95 \% \text { confidence } \\
\text { interval }\end{array}$} \\
\hline & Value & Lower & Upper \\
\hline Relative risk for TS & 5.531 & 2.806 & 10.903 \\
\hline No macroscopic ETE & 1.442 & 1.260 & 1.650 \\
\hline With macroscopic ETE & 0.261 & 0.147 & 0.463 \\
\hline
\end{tabular}

TS: tumor size; ETE: extrathyroidal extension.

Table 4. Surgery and type of recurrence

\begin{tabular}{|l|c|c|c|}
\hline \multicolumn{4}{|c|}{ Surgery and type of recurrence } \\
\hline Recurrence & Lobectomy & Total thyroidectomy & Total \\
\hline Biochemical & 0 & 1 & 1 \\
\hline Local & 0 & 2 & 2 \\
\hline $\begin{array}{l}\text { Other type of } \\
\text { recurrence }\end{array}$ & 0 & 5 & 5 \\
\hline $\begin{array}{l}\text { No } \\
\text { recurrence }\end{array}$ & 14 & 69 & 83 \\
\hline Total & 14 & 77 & 91 \\
\hline
\end{tabular}

Pearson's Chi-square test (3) $=1.595$.

Fisher's exact test $=1.184$.

$\mathrm{p}=0.661$.

second primary neoplasms, mostly represented by breast cancer, followed by cervical cancer and non-Hodgkin lymphoma. This is due to several reasons: 1 ) these patients usually have concurrent smaller tumors, incidentally found on PET/CT when studying their primary lesions; 2) presence of a bias, given that the study site is an oncological reference center, and 3) these type of malignancies might represent genetic syndromes, 
Table 5. Type of recurrence and tumor size

\begin{tabular}{|c|c|c|c|c|}
\hline \multicolumn{5}{|c|}{ Type of recurrence and tumor size } \\
\hline Recurrence & 0 to $1 \mathrm{~cm}$ & 1 to $2 \mathrm{~cm}$ & $>2 \mathrm{~cm}$ & Total \\
\hline Biochemical & 0 & 1 & 0 & 1 \\
\hline Local & 1 & 0 & 1 & 3 \\
\hline $\begin{array}{l}\text { Other type of } \\
\text { recurrence }\end{array}$ & 0 & 3 & 2 & 5 \\
\hline $\begin{array}{l}\text { No } \\
\text { recurrence }\end{array}$ & 26 & 34 & 23 & 83 \\
\hline Total & 28 & 34 & 29 & 91 \\
\hline
\end{tabular}

Table 6. Surgery and tumor size

\begin{tabular}{|l|c|c|c|}
\hline \multicolumn{4}{|c|}{ Surgery and tumor size } \\
\hline Size & Lobectomy & Thyroidectomy & Total \\
\hline 0 to $1 \mathrm{~cm}$ & 5 & 22 & 27 \\
\hline 1 to $2 \mathrm{~cm}$ & 7 & 31 & 38 \\
\hline$>2 \mathrm{~cm}$ & 2 & 24 & 26 \\
\hline Total & 14 & 77 & 91 \\
\hline
\end{tabular}

Pearson's Chi-square test (2) $=1.655$

Fisher's exact test $=1.669$.

$\mathrm{p}=0.437$.

such as Cowden's syndrome (in the case of breast cancer), which were not studied by the genetics department $^{13}$.

Consistently with the literature, among the 278 patients, a 5 -year disease-free survival of $71.58 \%$ was obtained, and only $28.42 \%$ of patients had persistence or relapse, out of which two died with tumor activity. Most of our stage I and II patients are alive without tumor activity, while in the literature, these patients have a 10-year survival of 97 to $100 \%$ reported. Patients at clinical stage III, together with patients who required an extended resection, represent the group of locally advanced cancer and all had tumor activity at the time of analysis; a 10-year survival of 88 to $95 \%$ has been described in this group. The percentage of patients with metastatic disease was consistent with previously-described figures $(2 \text { to } 5 \%)^{11,14}$.

The relapse rate is consistent with that reported in the literature, with $28.42 \%$, with most patients having extrathyroidal extension (87\%). Regarding relapses, the

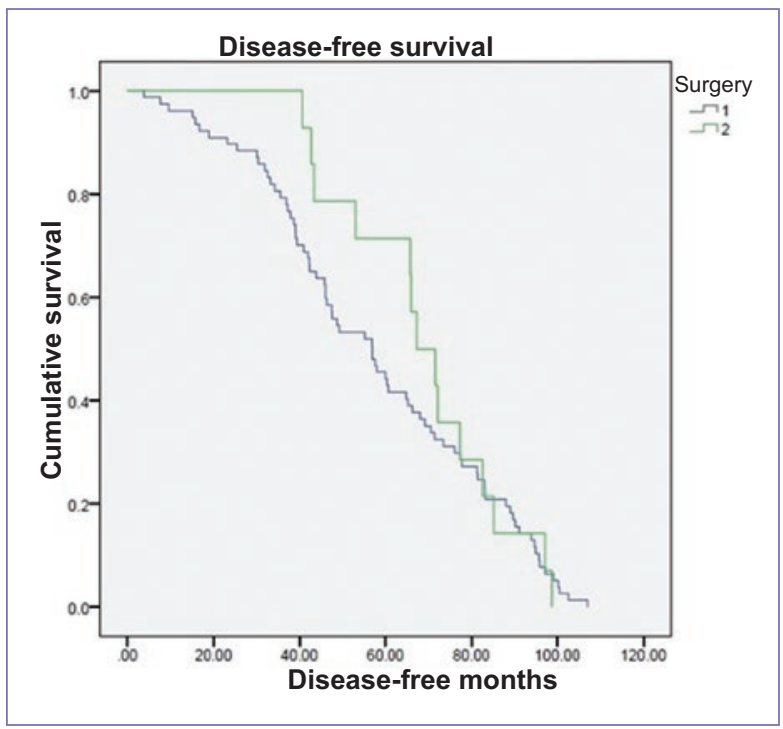

Figure 2. Mean disease-free survival (surgery - recurrence). Surgery: 1) total thyroidectomy, 57.97 months; lobectomy, 68.81 months.

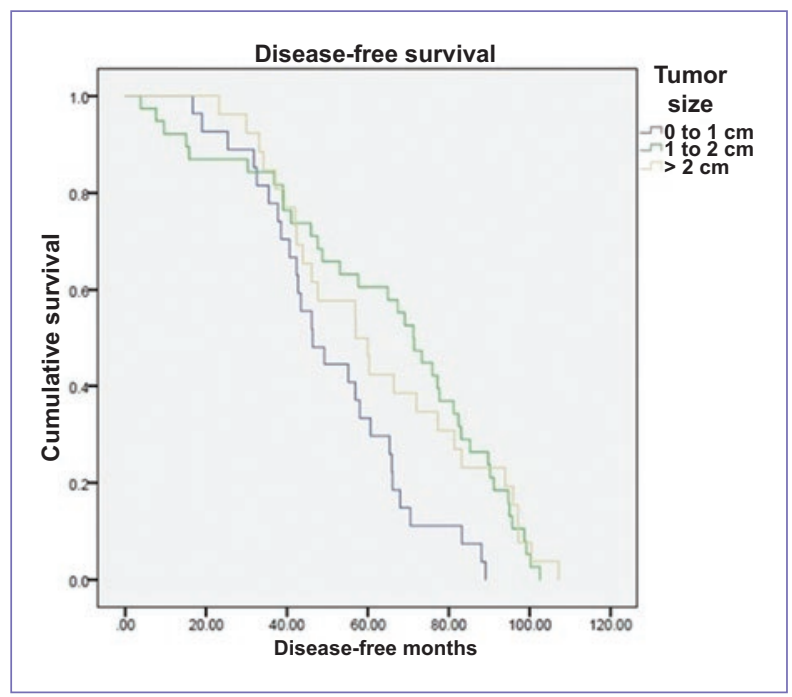

Figure 3. Mean recurrence-free survival (tumor size recurrence). 0 to $1 \mathrm{~cm}, 50.91$ months; 1 to $2 \mathrm{~cm}$, 63.89 months; > 2 cm, 62.49 months.

vast majority was regional and/or distant, followed by biochemical and local structural, with percentages very close to those reported $3,7,8,11$.

Regarding the percentage of $A D$ by risk groups, it was consistent with that reported by ATA, except for the high-risk group, where a higher percentage of almost twice as many patients without disease was found (63.2 vs. $32 \%)$; we consider that this finding might be 
due to lack of years of follow-up in the analysis. Relapse with structural disease in low-risk groups is 1 to $2 \%$, and in intermediate risk groups, $8 \%$, which is comparable to the findings of this series ${ }^{11}$.

Macroscopic extrathyroidal extension was found in 70 patients $(25.2 \%)$, the majority of which belonged to the 2 to $4-\mathrm{cm}$ group, out of which $64.78 \%$ are alive without tumor activity, which is comparable to the percentage of $A D$ among high-risk patients. When an association of risk with tumor size and macroscopic extrathyroidal extension is made, it corroborates that it becomes significant in tumors larger than $2 \mathrm{~cm}(35 \%)$, practically doubling the risk versus tumors smaller than $2 \mathrm{~cm}(15 \%) ;$ in other words: a patient with a tumor larger than $2 \mathrm{~cm}$ has a 5.5 -fold higher risk of having macroscopic extrathyroidal extension.

Optimal extent of surgery has always been controversial. Those who propose total thyroidectomy argue that it is associated with better local control and that it facilitates follow-up by ablation, which in turn allows serial $\mathrm{Tg}$ measurements. Other authors claim that selective lobectomy is a sufficient and adequate treatment for a group of patients with favorable prognostic characteristics and that systematic total thyroidectomy reoresents an over-treatment that exposes the patient to an unnecessary risk of morbidity and that there is no benefit associated with iodine administration, since these patients have an almost perfect survival.

Assuming that a group of patients are susceptible to appropriate treatment exclusively with lobectomy without increasing the risk of relapse or mortality, the characteristics of this group are controversial, especially with regard to tumor size. Recommendations have varied, from practicing it in all tumors smaller than $5 \mathrm{~cm}$ to avoiding it in all tumors larger than $1 \mathrm{~cm}$; however this recommendation never came from a specific study.

We theorize that tumor size is related to the risk of extrathyroidal extension and that it is susceptible to consistent ultrasound measurement in the preoperative period. If this is true, the tumor size chosen as a cut-off level for lobectomy should be associated with a low probability of macroscopic extrathyroidal extension, since this is associated with a high risk of local relapse. Therefore, it is necessary to administer adjuvant treatment to reduce risks, and this can only be efficaciously accomplished by eliminating all thyroid tissue. In addition, in order for only lobectomy to be performed, other conditions have to be be met, such as absence macroscopic multi-centric disease and macroscopic extrathyroidal extension within the preoperative or transoperative period, as well as regional or distant disease.
Our study seeks to define the tumor size at which significant risk of macroscopic extrathyroidal extension starts to exist. We found that from $2 \mathrm{~cm}$ on there is a higher than $35 \%$ risk, while with smaller sizes the risk does not exceed $13 \%$; therefore, we believe that only tumors smaller than $2 \mathrm{~cm}$ should be considered for lobectomy if they also meet the above indicated conditions.

We found that, in selected patients, lobectomy is not associated with a higher risk of local relapse in comparison with a group matched by the main prognostic factors and treated with total thyroidectomy. If a treatment obtains comparable results to those obtained with total thyroidectomy without exposing the patient to higher morbidity and costs, it is the best treatment.

Regarding the limitations of our study, first, it is a retrospective cohort study, which introduces biases that we tried to control by matching the patients using the main prognostic factors. In addition, it has a relatively limited number of patients and an average follow-up of 60 months or 5 years (range, 244 to 3,751 days), which could limit the identification of late events. Previous works have described that $30 \%$ of patients with thyroid cancer will develop some type of relapse over the course of decades, and that $66 \%$ occur within the first decade, out of which $74 \%$ are in cervical lymph nodes, $20 \%$ in the surgical bed and the trachea, and $6 \%$ in muscles, with the latter two resulting in death in $8 \%$ of patients; $21 \%$ will have distant recurrence, with the lung being the most commonly involved site in $63 \%$, and being the cause of death in $50 \%$ of patients with relapse, although current data document that relapses occur in earlier periods (2 to 5 years) $)^{7,8-10}$.

Our results suggest a clear relationship between tumor size and macroscopic extrathyroidal extension. This information opens the possibility of new studies. We consider that patients with tumors smaller than $2 \mathrm{~cm}$, without metastases (regional and/or distant) or macroscopic extrathyroidal extension or multicentrality, can be treated with lobectomy, since there is no higher risk of relapse. We believe that it is possible for similar oncological results to be obtained with lower morbidity using lobectomy, and, for this reason, it should be considered in a selected group of patients.

\section{Acknowledgements}

To my mother, whom I remember with love, to Dr. Martín Granados for his professional and personal teachings, and for his help in difficult times, to 
Dr. Alejandra Palafox for helping me arrive to the results, to the National Institute of Cancer for my training as a surgical oncologist and as a person. To doctors José Granell and Raimundo Gutiérrez for their support and teachings in Spain.

\section{References}

1. Grant CS, Hay ID, Gough IR, Bergstralh EJ, Goellner JR, McConahey WM Local recurrence in papillary thyroid carcinoma: is extent of surgical resection important? Surgery. 1988;104:954-62.

2. Mazzaferri EL, Kloos RT. Clinical review 128: Current approaches to primary therapy for papillary and follicular thyroid cancer. J Clin Endocrinol Metab. 2001:86:1447-63.

3. Nixon IJ, Ganly I, Patel SG, Palmer FL, Whitcher MM, Tuttle RM, et al Thyroid lobectomy for treatment of well differentiated intrathyroid malig nancy. Surgery. 2012;151:571-9.

4. Vaisman F, Shaha A, Fish S, Michael Tuttle R. Initial therapy with either thyroid lobectomy or total thyroidectomy without radioactive iodine remnant ablation is associated with very low rates of structural disease recurrence in properly selected patients with differentiated thyroid cancer. Clin Endocrinol (Oxf). 2011;75:112-9.

5. Barney BM, Hitchcock YJ, Sharma P, Shrieve DC, Tward JD. Overall and cause-specific survival for patients undergoing lobectomy, near-total, or total thyroidectomy for differentiated thyroid cancer. Head Neck. 2011;33:645-9.
6. Mendelsohn AH, Elashoff DA, Abemayor E, St John MA. Surgery for papillary thyroid carcinoma: is lobectomy enough? Arch Otolaryngol Head Neck Surg. 2010;136:1055-61.

7. Mazzaferri EL, Jhiang SM. Long-term impact of initial surgical and medical therapy on papillary and follicular thyroid cancer. Am J Med. 1994; 97:418-28.

8. American Thyroid Association (ATA) Guidelines Taskforce on Thyroid Nodules and Differentiated Thyroid Cancer, Cooper DS, Doherty GM, Haugen BR, Kloos RT, Lee SL, et al. Revised American Thyroid Association management guidelines for patients with thyroid nodules and differentiated thyroid cancer. Thyroid. 2009;19:1167-214.

9. Leenhardt L, Erdogan MF, Hegedus L, et al. European thyroid association guidelines for cervical ultrasound scan and ultrasound-guided techniques in the postoperative management of patients with thyroid cancer. Eur Thyroid J. 2013;2:147-59.

10. Haugen BR, Sawka AM, Alexander EK, et al. American Thyroid Association Guidelines on the Management of Thyroid Nodules and Differentiated Thyroid Cancer Task Force Review and Recommendation on the Proposed Renaming of Encapsulated Follicular Variant Papillary Thyroid Carcinoma Without Invasion to. Thyroid. 2017;27(4):481-3.

11. National Cancer Institute 2009 [Internet]. Horner, Marie-Joseph. SEER Cancer Statistics Review 1975-2006. Disponible en: http://seer.cancer. gov/csr/1975_2006/.

12. Hay ID, Grant CS, van Heerden JA, et al. Papillary thyroid microcarcinoma: a study of 535 cases observed in a 50 -year period. Surgery. 1992;112(6):1139-46.

13. Globocan 2018. Mexico [Internet]. World Health Organization, International Agency for Research on Cancer; 2018. Disponible en: http://gco.iarc. $\mathrm{fr} /$ today/data/factsheets/populations/484-mexico-fact-sheets.pdf.

14. Gress, Donna M, Edge, et al. Principles of cancer staging. AJCC Cancer Staging Manual. 8th ed. New York: Springer; 2017. 